\title{
A Hybrid Model in Comparing the Performance of Wired and Wireless Technologies by Using the Markov Algorithm and Queuing Petri Nets
}

\author{
V.B.Kirubanand \\ Department Of Mca, \\ Sri Krishna College Of Engineering And \\ Technology \\ Coimbatore-641008.
}

\author{
Dr.S.Palaniammal \\ Department Of Science And Humanities, \\ Vlb Janakiammal College Of Eng And Tech \\ Coimbatore-641042
}

\begin{abstract}
The main theme of this paper is to find the performance of using the wired and the wireless devices such as Hub, Switch, Bluetooth, Wi-Fi and Wimax. Queueing Petri net model and Markov algorithm with the security of Huffman algorithm were used in performance analysis. This paper mainly focuses on comparison of the different Wired and Wireless technologies in terms of inter-arrival time and Inter-service time by using Markov algorithm $(\mathrm{M} / \mathrm{M}(\mathrm{a}, \mathrm{b}) / 1)$. The inter -arrival and Interservice time were obtained using the Poisson distribution. By comparing the service rates of the Wired and Wireless technologies it has been found that the service rate offered by the Wireless technologies were very efficient for implementation and the values obtained from the Wimax technology is very much efficient that other wireless technologies. The performance of all the Wireless technologies can be obtained by using the value of the Bluetooth technology as the other wireless devices are not in prevalence. The performance is calculated in terms of Expected Waiting time and Expected Busy period. Queueing Petri Nets were used to facilitate the integration of both hardware and software aspects of the system behavior in the Hybrid model. The purpose of Huffman algorithm in Steganography is to send the hidden information from one system to another system through Wired or Wireless devices. Queueing Petri Net are very powerful as a performance analysis and prediction tool. Thus Queueing Petri Net and Markov algorithm can be used in the fore coming networking technologies to obtain better performanceresults.
\end{abstract}

Keywords: Queueing Petri-nets, Markov algorithm, Bulk service, Distribution value, Inter-Arrival time, Inter-Service time, Steganography.

\section{INTRODUCTION \\ HUB}

A network hub or repeater hub is a device for connecting multiple twisted pair or fiber optic Ethernet devices together and thus making them act as a single network segment. Hubs work at the physical layer (layer 1) of the OSI model. The device is thus a form of multiport repeater. Repeater hubs also participate in collision detection, forwarding a jam signal to all ports if it detects a collision

\section{SWITCH}

A network switch is a small hardware device that joins multiple computers together within one local area network (LAN).
Technically, network switches operate at layer two (Data Link Layer) of the OSI model.

\section{BLUETOOTH TECHNOLOGY}

Bluetooth is an open wireless protocol for exchanging data over short distances from fixed and mobile devices, creating personal area networks (PANs). It was originally conceived as a wireless alternative to RS232 data cables. It can connect several devices, overcoming problems of synchronization. The implication is that Bluetooth does the same with communications protocols, uniting them into one universal standard. Bluetooth uses a radio technology called frequency-hopping spread spectrum, which chops up the data being sent and transmits chunks of it on up to 79 frequencies. In its basic mode, the modulation is Gaussian frequency-shift keying (GFSK). It can achieve a gross data rate of $1 \mathrm{Mb} / \mathrm{s}$. Bluetooth provides a way to connect and exchange information between devices such as mobile phones, telephones, laptops, personal computers, printers, Global Positioning System (GPS) receivers, digital cameras, and video game consoles through a secure, globally unlicensed Industrial, Scientific and Medical (ISM) $2.4 \mathrm{GHz}$ short-range radio frequency bandwidth. The Bluetooth specifications are developed and licensed by the Bluetooth Special Interest Group (SIG). The Bluetooth SIG consists of companies in the areas of telecommunication, computing, networking, and consumer electronics

\section{WI-FI TECHNOLOGY}

The name of a popular wireless networking technology that uses radio waves to provide wireless high-speed Internet and network connections. The Wi-Fi Alliance, the organization that owns the Wi-Fi (registered trademark) term specifically defines $\mathrm{Wi}-\mathrm{Fi}$ as any "wireless local area network (WLAN) products that are based on the Institute of Electrical and Electronics Engineers' (IEEE) 802.11 standards."

Initially, Wi-Fi was used in place of only the $2.4 \mathrm{GHz} 802.11 \mathrm{~b}$ standard, however the Wi-Fi Alliance has expanded the generic use of the Wi-Fi term to include any type of network or WLAN product based on any of the 802.11 standards, including $802.11 \mathrm{~b}, 802.11 \mathrm{a}$, dual-band, and so on, in an attempt to stop confusion about wireless LAN interoperability.

Wi-Fi works with no physical wired connection between sender and receiver by using radio frequency (RF) technology, a frequency within the electromagnetic spectrum associated with radio wave propagation. When an RF current is supplied to an 
antenna, an electromagnetic field is created that then is able to propagate through space. The cornerstone of any wireless network is an access point (AP). The primary job of an access point is to broadcast a wireless signal that computers can detect and "tune" into. In order to connect to an access point and join a wireless network, computers and devices must be equipped with wireless network adapters.

\section{WIMAX TECHNOLOGY}

Wimax can be used for wireless networking like the popular WiFi. Wimax, a second-generation protocol, allows higher data rates over longer distances, efficient use of bandwidth, and avoids interference almost at minimum. Wimax can also be used in Automatic teller machines, Online gaming, Multimedia communication, Medical applications, Vehicular data and voice, Sensor networks, Telematics and telemetry. Mobile transmission of information in emergency situations, Real-time monitoring, alerting, and controlling the process of dangerous works.

\section{IEEE Standards:}

WiMax is based on IEEE 802.16.

\section{Range:}

WiMAX antenna is expected to have a range of up to 40 miles with speeds of $70 \mathrm{Mbps}$ or more.

\section{Scalability:}

WiMax is designed to efficiently support from one to hundreds of Consumer premises equipments (CPE)s, with unlimited subscribers behind each CPE. Flexible channel sizes from $1.5 \mathrm{MHz}$ to $20 \mathrm{MHz}$.

\section{Bit rate:}

Wi-Fi works at $2.7 \mathrm{bps} / \mathrm{Hz}$ and can peak up to $54 \mathrm{Mbps}$ in 20 $\mathrm{MHz}$ channel. But other technologies like WiMax works at $5 \mathrm{bps} / \mathrm{Hz}$ and can peak up to $100 \mathrm{Mbps}$ in a $20 \mathrm{MHz}$ channel

\section{Quality of service:}

Other wireless technology does not guarantee any QoS but WiMax provide several level of QoS. Also WiMax can bring the underlying Internet Connection needed to service other local wireless networks

The main objective of these models is to find the following with the usage of Markov algorithm

\section{- To find the waiting time.}

- To find the busy period.

\section{STEGANOGRAPHY}

Steganography is the art of hiding information in images, sounds or any other file format than the routine format. A piece of data or information hidden into a image or sound file is extremely difficult to catch and this can lead to vast propagation of the material through internet or other media. Steg-Analysis and decryption techniques are applied to get the data back to its original form.[1]

\section{QUEUEING NETWORKS}

A network consists of a set of interconnected queues. Each queue represents a service station, which serves requests (also called jobs) sent by customers. A service station consists of one or more servers and a waiting area which holds requests waiting to be served. When a request arrives at a service station, its service begins immediately if a free server is available. Otherwise, the request is forced to wait in the waiting area. Service is done as per the general bulk service rule introduced by Neuts(1967) with $a=1$. As per this rule immediately after the completion of the service, if the server finds a unit present, it start its service; if it finds one or more but almost bit takes them all in a batch and if it finds more than bit takes in the batch for service $b$ units, while others wait. The batch takes a minimum of one unit and a maximum of $b$ units.[6]The time between successive request arrivals is called interarrival time. Each request demands a certain amount of service, which is specified by the length of time a server is occupied serving it, i.e the service time. The delay is the amount of time the request waits in the waiting area before its service begins. The response time is the total amount of time the request spends at the service station, i.e. the sum of the delay and the service time.

\section{PETRI NETS}

Petri Nets were introduced in 1962 by Carl Adam Petri. An ordinary Petri Net (also called Place-Transition Net) is a bipartite directed graph composed of places, drawn as circles, and transitions, drawn as bars.

\section{BASIC QUEUEING PETRI NETS}

The main idea in the creation of the QPN formalism was to add and timing aspects to the places of Colored Generalized Stochastic Petri Nets.[3] This is done by allowing queues (service stations) to be integrated into places of CGSPNs. A place of a CGSPN which has an integrated queue is called a place and consists of two components, the queue and a depository for tokens which have completed their service at the queue. This is depicted in Figure 3.

The behavior of the net is as follows: tokens, when fired into a place by any of its input transitions, are inserted into the queue according to the queue's scheduling strategy. Tokens in the queue are not available for output transitions of the place. After completion of its service, a token is immediately moved to the depository, where it becomes available for output transitions of the place. This type of place is called a timed place. Tokens in immediate places can be viewed as being served immediately. Scheduling in such places has priority over scheduling/service in timed places and firing of timed transitions.

\section{HIERARCHICAL QUEUEING PETRI NETS}

As already mentioned the main hurdle to the quantitative analysis of QPN's is the fact that most analysis techniques available are based on Markov Chains and are therefore susceptible to the state space explosion problem. More specifically, as one increases the number of queues and tokens in a QPN, the size of the state space of the underlying Markov Chain grows exponentially and quickly exceeds the capacity of today's computers. This imposes a limit on the size and complexity of the models that are analyzable. An attempt to 
alleviate this problem was the introduction of HierarchicallyCombined Queueing Petri Nets (HQPNs)[4]. The main idea is to allow hierarchical model specification and then exploit the hierarchical structure for efficient numerical analysis. This type of analysis is termed structured analysis and it allows models to be solved, which are about an order of magnitude larger than those analyzable with conventional techniques.

HQPNs are a natural generalization of the original QPN formalism. In HQPNs a place may contain a whole QPN instead of a single queue. Such a place is

Every subnet of a HQPN has a dedicated input and output place, which are ordinary places of a colored Petri Net. Tokens being inserted into a subnet place after a transition ${ }^{-}$ring are added to the input place of the corresponding HQPN subnet. The semantics of the output place of a subnet place is similar to the semantics of the depository of a place: tokens in the output place are available for the output transitions of the subnet place. Tokens contained in all other places of the HQPN subnet are not available for the output transitions of the subnet place. Every HQPN subnet also contains actual i population place, which is used to keep track of the total number of tokens fired into the subnet place.

\section{MARKOV ALGORITHM}

A Markov algorithm is a string rewriting system that uses grammar-like rules to operate on strings of symbols. Markov algorithms have been shown to be Turing-complete, which means that they are suitable as a general model of computation and can represent any mathematical expression from its simple notation.[5]

\section{BULK SERVICE RULES}

There could be a number of a policies or rules according to which batches for bulk service may be formed. The following are the types of bulk service policies or rules frequently discussed in the literature.

Bailey (1954) and Downton (1955) considers that units are served in batches of not more than b (say).If, immediately after the completion of a service, the server finds more than $b$ units waiting, he takes a batch of $b$ units for service while others wait ;if he finds $r$ units $(0 \leq r \leq b)$, he takes all he $r$ units in a batch for service . Bloemena (1960), Jaiswal(1960) Neuts (1967) consider the same rule with the restriction that $r !=0 \quad(1 \leq r \leq b)$, i.e. the service facility stops until a unit arrives. This rule will be called the usual bulk service rule, while bailey's rule will be its modified type ( it will be also called bulk service rule with intermittently available server). Jaiswal points out that the distribution of the queue length for the modified rule can be obtained from that of the usual rule.[6].

The rule with a fixed batch size ' $\mathrm{k}$ ' has been considered by Fabens (1961), Takacs (1962) and others . In this case the server waits until there are $\mathrm{k}$ units, and serves all the $\mathrm{k}$ units in a batch. If there are more than $\mathrm{k}$ waiting when the server becomes free, he takes a batch $\mathrm{k}$ for service, while others wait.

Neuts (1967) considers the general bulk service rule: if, immediately after the completion of a service, the server finds less than a units present, he waits until there are a units, whereupon he takes the batch of a units for service; if he finds a or more but atmost b,he takes them all in a batch and if he finds more than $b$,he takes in the batch for service $b$ units, while others wait. The batch takes a minimum of a units and a maximum of $b$ units. This rule will be called general bulk service rule as rules under (1) and (2) can be taken as particular cases.

Bhat (1963) considers the rule that the number taken in a batch is a random variable $\mathrm{y}$. The corresponding Markov model is denoted by $\mathrm{M} / \mathrm{M}^{\wedge}(\mathrm{y}) / 1$.

There are actual situations where one rule seems to be better suited or to be more appropriate than the others

\section{Performance measures}

(i) Probability that the server is idle

$p_{0,0}=\frac{(1-r)}{1-r+(\lambda / \mu)}=\frac{(1-r)^{2}}{(1-r)^{2}+r\left(1-r^{b}\right)}$,

(ii) Probability that the server is busy and $n$ units in the system

$p_{1, n}=\frac{(1-r)\left(1-r^{b}\right)}{(1-r)^{2}+\left(1-r^{b}\right)} r^{n+1}, \mathrm{n}=0,1,2, .$.

(iii)Waiting Time Density

$\mathrm{v}(\mathrm{t})=\frac{\lambda_{p 0,0}}{\left(c_{1}(-\mathrm{r})\right)}\left[\left(1-r^{b}\right) \exp \left\{-\mu\left(1-r^{b}\right) \mathrm{t}\right\}\right]$

(iv)Expected Waiting Time Density

$\mathrm{E}(\mathrm{T})=\frac{r}{\mu\left[(1-r)^{b}+r\left(1-r^{b}\right)\right]}$

(v)Expected Busy Period

$\mathrm{E}(\mathrm{B})=\frac{1}{\mu(1-r)}$

From the above formulas we are calculating the Performance of Client-Server model

\section{Distribution Value}

\begin{tabular}{|c|c|c|c|c|c|}
\hline TECHNOLOGIES & REQ/RES & $\begin{array}{c}\mathbf{1}^{\mathrm{st}} \\
\text { System }\end{array}$ & $\begin{array}{c}2^{\text {nd }} \\
\text { System }\end{array}$ & $\begin{array}{c}3^{\text {rd }} \\
\text { System }\end{array}$ & $\begin{array}{l}\text { Inter- } \\
\text { arrival / } \\
\text { Inter- } \\
\text { service }\end{array}$ \\
\hline HUB & $\begin{array}{l}\text { Request } \\
\text { Response }\end{array}$ & $\begin{array}{l}0.480 \\
3.900\end{array}$ & $\begin{array}{l}0.560 \\
4.970\end{array}$ & $\begin{array}{l}0.670 \\
6.030\end{array}$ & $\begin{array}{c}1 / 0.5700 \\
1 / 4.9670\end{array}$ \\
\hline SWITCH & $\begin{array}{l}\text { Request } \\
\text { Response }\end{array}$ & $\begin{array}{l}0.300 \\
2.800\end{array}$ & $\begin{array}{l}0.340 \\
3.650\end{array}$ & $\begin{array}{l}0.400 \\
4.700\end{array}$ & $\begin{array}{l}1 / 0.3400 \\
1 / 3.7170\end{array}$ \\
\hline BLUETOOTH & $\begin{array}{l}\text { Request } \\
\text { Response }\end{array}$ & $\begin{array}{l}0.280 \\
2.530\end{array}$ & $\begin{array}{c}0.31 \\
3.230\end{array}$ & $\begin{array}{l}0.360 \\
4.310\end{array}$ & $\begin{array}{l}1 / 0.3170 \\
1 / 3.3570\end{array}$ \\
\hline WIFI & $\begin{array}{l}\text { Request } \\
\text { Response }\end{array}$ & $\begin{array}{l}0.220 \\
2.360\end{array}$ & $\begin{array}{l}0.271 \\
2.980\end{array}$ & $\begin{array}{l}0.338 \\
3.780\end{array}$ & $\begin{array}{l}1 / 0.2760 \\
1 / 3.0400\end{array}$ \\
\hline
\end{tabular}




\section{Inter-Arrival and Inter-Service timing}

\begin{tabular}{|r|l|l|l|l|}
\hline \multicolumn{1}{|l|}{ TECHNOLO- } & REQUEST & RESPONS & $\mathbf{E}(\mathbf{T})$ & $\mathbf{E}(\mathbf{B})$ \\
& $(\lambda)$ & E $(\mu)$ & & \\
\hline HUB & $1 / 0.5700$ & $1 / 4.9670$ & $\mathbf{0 . 7 0 5 5 8}$ & $\mathbf{5 . 6 1 1 9}$ \\
& & & & \\
\hline SWITCH & $1 / 0.3400$ & $1 / 3.7170$ & $\mathbf{0 . 4 0 4 3 2}$ & $\mathbf{4 . 0 8 4 2}$ \\
& & & & \\
\hline BLUETOOTH & $1 / 0.3170$ & $1 / 3.3570$ & $\mathbf{0 . 3 6 2 9}$ & $\mathbf{3 . 6 9 4 1}$ \\
& & & & \\
\hline WIFI & $1 / 0.2760$ & $1 / 3.0400$ & $\mathbf{0 . 3 2 7 3 3}$ & $\mathbf{3 . 4 3 7 1}$ \\
& & & & \\
& & & & \\
\hline
\end{tabular}

A comparative study of the wired technology with the wireless technology has been done with help of the markovian model $\mathrm{M} / \mathrm{M}(1, \mathrm{~b}) / 1$ with the bandwidth of $100 \mathrm{mbps}$.

The probability that the server is idle and the probability that the server is busy are same for both of the networks.

$\lambda$ - Arrival rate, E(T) - Expected waiting time, $\mu$ - Service rate, E (B) - Expected busy period.

\section{CONCLUSION}

Thus this Hybrid model research work concludes that the performance of the client server architecture is better in the Wimax technology when compared to Hub network, switch network, Bluetooth, and Wi-Fi. We hope to motivate further research in this area.

\section{REFERENCES}

[1] www.bluetooth.com

[2] G. Bolch, S. Greiner, H. De Meer, and K. S. Trivedi. Queueing Networks and Markov Chains - Modeling and Performance Evaluation with Computer Science Applications. Wiley, NewYork, 1998.
[3]F. Bause and F. Kritzinger. Stochastic Petri Nets -An Introduction to the Theory. Vieweg Verlag, 2002.

[4]F. Bause, P. Buchholz, and P. Kemper. Hierarchically Combined Queueing Petri Nets. In Proc. of 11th Intl. Conference on Analysis and Optimization of Systems, Discrete event Systems, Sophie-Antipolis (France), 1994.

[5]http://www.knowledgerush.com/kr/encyclopedia/markov_alg orithm/

[6] Stochastic processes. Second edition. J.Medhi. New Age International Publishers.

[7] performance modeling of distributed e-business application using queueing petri nets.By-Kounev,S.;Buchmann,A.,2003

[8]http://3g4g.blogspot.com/2008/01/heard-of-bluetooth.html

[9]www.wikipedia.com

[10]www.webopedia.com

[11]www.Wi-fi.com

[12]www.wimax.com

[13]www.hybrid.com

V.B.Kirubanand received the M.C.A. degree from Erode Arts \& Science College, Erode in 1996.He also obtained his M.Phil at Periyar university in 2008 and at present he is pursuing his $\mathrm{PhD}$ in Anna University, Coimbatore. He worked as lecturer, senior lecturer, Instructor and as Head of the Department in various colleges from 1996 to 2010 and presently he is working as Asst Prof, Master of Computer Applications Department, Sri Krishna College of Engineering and Technology, Coimbatore. He has published 21 books regarding computer application and software's. His area of interest includes network security and wireless networks.

Dr.S.Palaniammal received her doctoral degree in applied mathematics from PSG Technology, Coimbatore in 2006. She is currently working as Professor and Head in the department of Science and Humanities at VLB Janakiammal College of Engineering and Technology, Coimbatore. She has also published 18 papers in international and national conferences. Her area of interest is Queueing Theory, Image Processing and Networking. Under her guidance 9 scholars are doing research work and at resent one M.Phil has been completed. She has authored 7 books in Mathematics for B.E/B.Tech students. 